\title{
Publisher's Note: Evidence of a fractional quantum Hall nematic phase in a microscopic model [Phys. Rev. B 96, 035150 (2017)]
}

\author{
N. Regnault, J. Maciejko, S. A. Kivelson, and S. L. Sondhi
}

(Received 19 September 2017; published 25 September 2017)

DOI: 10.1103/PhysRevB.96.119910

This paper was published online on 27 July 2017 with an error in the Acknowledgments. The last sentence of the Acknowledgments on page 6 should read as "S.A.K. was supported by NSF-DMR via Grant No. 1608055, and S.L.S. was supported by NSF-DMR via Grant No. 1311781." The Acknowledgments have been corrected as of 18 September 2017. The Acknowledgments are incorrect in the printed version of the journal. 